near the equator, such as Lake Titicaca. In contrast to most other parts of the world, few of these waters have been much affected by man and this is reflected in an outburst of speciation. There are well over 600 species of fish in the entire area, though this is likely to prove conservative since it is not yet known precisely how many fish species there are in the Amazonian system alone. A good start has been made with the publication of the record of Argentinian fish.

The meeting drew up a series of seven resolutions to put this situation to rights. The mood and emphasis were essentially practical. First an inventory of Latin American hydrobiologists is to be compiled, with their special interests. An association of hydrobiologists is to be set up. There is to be a register of outside experts who are prepared to act as consultants and run training courses at the universities (the lack of trained manpower is "fantastic", comments a European participant). Interchange of technical staff within Latin America is to be promoted. Documentation is to be centred on a single information centre in Latin America which may also publish a periodical and run an advisory service. Bilateral research projects are to be pursued such as that currently going on in Brazil where German scientists are at work. There are to be joint (multinational) projects on pollution in fresh waters and on fish culture in Latin America.

\section{Results from Ariel 3}

The Ariel 3 satellite, launched on May 5 last year, seems, in the main, to have been a success. This was clear from a meeting held at the Royal Society on April 24 at which preliminary scientific results from the satellite were discussed. It is particularly pleasing that the power supply and data handling systems have functioned perfectly in Ariel 3, the first satellite to be designed and built entirely in the United Kingdom. It was launched by an American Scout rocket into a near Earth orbit having a perigee of $494 \mathrm{~km}$ and an apogee of $600 \mathrm{~km}$ from the Western Test Range in California, and has produced on the average 6 hours of data for each day in orbit. Five space research groups have experiments on board-Birmingham University, Manchester University (Jodrell Bank), Sheffield University, the SRC Radio and Space Research Station, and the Meteorological Office.

The success of the satellite is qualified, however, because interference from the Birmingham experiments has limited the usefulness of the Jodrell Bank experiment which investigates cosmic and ionospheric radio noise. The Birmingham experiment is designed to measure the number and temperature of electrons in the high regions of the ionosphere through which the satellite passes. This it does by means of sensors fixed to the ends of two of the satellite's four solar cell booms. Signals applied to the sensors as part of the Birmingham experiment are picked up by the Jodrell Bank radio aerial, a loop of wire strung round the extremities of the satellite's booms. The Jodrell Bank experiment was designed to measure the intensity of cosmic radio noise in the range $2-4 \cdot 2 \mathrm{MHz}$, part of the radio spectrum which cannot penetrate the ionosphere, and to investigate noise bands generated in the ionosphere. At the meeting, Professor F. G. Smith and Mr P. Gregory of Jodrell Bank explained that the interference was caused by an unexpected coupling effect through the ionosphere outside Ariel 3. This means that the Jodrell Bank results have to be sorted from the unwanted signal; nevertheless the data are giving values of cosmic radio noise at $4.25 \mathrm{MHz}$, which the Jodrell Bank group hopes to extend to the remainder of the range.

The Sheffield experiment measures very low frequency radio waves at three frequencies, $3 \cdot 2,9 \cdot 6$ and $16 \mathrm{kHz}$, and is investigating naturally occurring very low frequency phenomena such as whistlers, hiss and chorus, as well as transmissions from the Rugby Rarlio Station GBR at $16 \mathrm{kHz}$ and from an Australian station at $15.5 \mathrm{kHz}$. Although the Sheffield experiment also incorporates a loop aerial adjacent to the Jodrell Bank loop, the Sheffield experimenters report no interference from other equipment in the spacecraft. Measurements of chorus and hiss signals produced by energetic charged particles in the upper atmosphere, should yield information about interactions between energetic particles and radio waves. World-wide maps of the signal strength of transmissions from very low frequency radio stations are giving information about the way these radio waves are guided through the atmosphere.

The experiment designed by the Radio and Space Research Station at Slough, on the other hand, is more concerned with events lower in the atmosphere. This experiment measures the intensity of radio noise from lightning discharges in a defined area over which the satellite is passing. Receivers measure terrestrial radio noise at six frequencies, but the experimenters say that signals from a transmitter on the ground, which started operating after Ariel 3 was launched, interfere severely with one of the channels. So far, the group has concentrated on analysing data from the European area, but they say there is evidence that tropical lightning discharges are less potent generators of high frequency radio noise than discharges in tem. perate regions.

The fifth experiment, designed by the Meteorological Office, measured the amount of oxygen in the atmosphere between heights of 150 and $250 \mathrm{~km}$ above the Earth. Because this region is too high to be studied by small rockets but is below the orbiting heights of satellites, it has not been studied extensively. The predicted lifetime of the experiment was short, how. ever, because the gas in its detectors was expected to be destroyed by solar radiation. The experiment worked by measuring the attenuation of sunlight as the satellite passed into the Earth's shadow. At this time sunlight illuminating the satellite has to pass through low levels of the atmosphere, where certain wavelengths in the ultraviolet are strongly absorbed by oxygen. During the few weeks that the experiment functioned it made measurements over a range of latitudes; these are still being studied.

\section{Anglo-Australian Telescope from our Astronomy Correspondent}

The $150 \mathrm{in}$. telescope to be built at the Siding Spring Observatory of the Australian National University is to be a copy of the 150 in. telescope now under construction at the Kitt Peak Observatory in Arizona. Only a few minor changes are to be made and these should simplify the construction and operation of the telescope. The result is that design time has been cut 